\title{
Resuscitation with Intact Cord Reduces the Rate of Asphyxia at 5 Minutes
}

\author{
Lixia Zheng, RM ${ }^{1}$ and Hong-yu Zhang ${ }^{2 *}$ \\ ${ }^{1}$ Department of Delivery Unit, Laiwu People's Hospital, Laiwu City, People's Republic of China \\ ${ }^{2}$ Department of Midwifery Education, Hainan Medical University, People's Republic of China
}

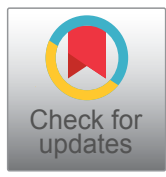

*Corresponding author: Hongyu Zhang, Department of Midwifery, Hainan Medical University, Haikou, 571119, China, Tel: $+860898-66892700$

\begin{abstract}
Objectives: Explore the effect of resuscitation with intact cord in neonate asphyxia.

Methods and materials: Retrospective cohort study. Asphyxia babes 104 cases from Jan. 2011 to Dec. 2013 in the seconde maternal and neonate hospital in Jinan Shandong province received immediate cord clamping and transferring to the emergence-bed for the resuscitation process were enrolled as controlled group; experimental group were those asphyxia babes 94 cases from Jan. 2014 to Dec. 2016 where they were resuscitated with intact cord in delivery bed. Data was recorded from the clinical record in the hospital. Information including maternal condition, delivery process and neonate condition were recorded. Following up were through the telephone for the neonate survival condition.
\end{abstract}

Results: The general information of maternal age, pregnant weeks, neonate birth weight in two groups was not of significant. The rate of asphyxia in two groups were not of statistical difference $(\chi 2=2.043 \mathrm{P}=0.153$; asphyxia rate at five minute in experimental group $4.3 \%(4 / 94)$ was lower than that in controlled group $13.5 \%(14 / 104$, of statistical difference $(\chi 2=4.968, P=0.026)$. Neonatal death and paralysis in two groups were not of statistical difference.

Conclusions: Resuscitation with intact cord reduces the rate of asphyxia at five minutes without increasing the neonate death and paralysis rate. It should be considered against the protocol of cutting the cord immediately and transferred to emergence bed.

\section{Keywords}

Neonate asphyxia, Resuscitations of neonate asphyxia, Delayed cord clamping, Neonate paralysis

\section{Introduction}

The benefit of delayed cord clamping till the pulsation of the cord ceased has been proved both to mothers and babies, as increased hemoglobin levels and iron store of babies at first 6 months $[1,2]$. The traditional method of cutting the cord immediately after birth has been abended from the routing clinical practice protocols [3].

And most of the recommendation on these issues supporting the delayed cord clamping on the normal babies. But for the asphyxia babies, argues remain for the keeping warm by transferring to the radiators and resuscitation on this table, that will cut the cord and this may be deprived the asphyxia babies the oxygen and nutrient supplies from the placenta while their lungs not start to work properly. Brouwer $E$ to measure the $\mathrm{PH}$ state from preterm babies with intact cord founded that median $\mathrm{SpO}_{2}$ of the infants increased quickly in the first $4 \mathrm{~min}$ after birth to a stable level above $90 \%$, that suggested placenta transfusion with intact cord improved neonate's conditions [4].

We have applied delayed cord clamping on resuscitation of asphyxia babies since 2014, and 94 cases asphyxia babies recovered and survived without palsy or other complications that offered good news to supporting the delayed cord clamping on asphyxia babies. We compared those case with data of asphyxia babies that were resuscitation in traditional methods that cutting cord and transferred to the bedsides radiator tables.

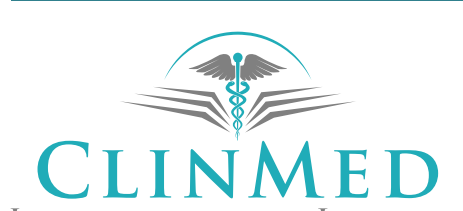

INTERNATIONAL LIBRARY
Citation: Zheng L, Zhang H (2021) Resuscitation with Intact Cord Reduces the Rate of Asphyxia at 5 Minutes. Obstet Gynecol Cases Rev 8:214. doi.org/10.23937/2377-9004/1410214

Accepted: November 13, 2021: Published: November 15, 2021

Copyright: (c) 2021 Zheng L, et al. This is an open-access article distributed under the terms of the Creative Commons Attribution License, which permits unrestricted use, distribution, and reproduction in any medium, provided the original author and source are credited. 
Table 1: Comparation of maternal and neonatal conditions between two groups.

\begin{tabular}{|l|l|l|l|l|}
\hline Indicators & $\begin{array}{l}\text { Resuscitation at radiator with } \\
\text { cutting cord immediately (104) }\end{array}$ & $\begin{array}{l}\text { Resuscitation at delivery } \\
\text { table with intact cord (94) }\end{array}$ & $\boldsymbol{t}$ // \\
\hline Maternal age (year) & $29.14 \pm 5.83$ & $30.93 \pm 5.85$ & 1.105 & 0.057 \\
\hline Pregnant (wks) & $38.17 \pm 3.90$ & $38.54 \pm 3.39$ & 0.683 & 0.496 \\
\hline Maternal complications & $4(4.2)$ & $4(4.3)$ & 0.000 & 0.988 \\
\hline Birth weight of newborns (g) & $3072.84 \pm 878.45$ & $3155.90 \pm 841.89$ & 0.664 & 0.508 \\
\hline Gender (female) & $37(38.9)$ & $41(43.6)$ & 0.425 & 0.514 \\
\hline Preterm (\%) & $19(54.3)$ & $16(45.7)$ & 0.278 & 0.598 \\
\hline Asphyxia at 1 min (\%) & $43(47.3)$ & $48(52.7)$ & 2.043 & 0.153 \\
\hline Asphyxia at 5 min (\%) & $14(13.3)$ & $4(4.3)$ & 4.968 & 0.026 \\
\hline Neonate death & $2(2.1)$ & $1(1.1)$ & 0.328 & 0.567 \\
\hline Palsy & $1(1.1)$ & $0(0.0)$ & Fisher & 1.000 \\
\hline
\end{tabular}

\section{Materials and Methods}

Cohort studies were carried out in JINAN Second maternal and neonatal hospital. Experimental groups were 94 cases asphyxia babies application of resuscitation with intact cord since Jan. in 2014 to Dec. 2016. Controlled group were those 104 cases resuscitation in traditional ways that cutting the cord immediately and transfer to radiator from Jan. 2011 to Dec. 2013. Data information was recorded form clinical records from the hospital. Research plan was approved by ethical committee from the hospital. We did not ask for each patient's approval. The geography information of mothers as pregnant state, complications during pregnant and birth, birth style, process of labor, postpartum hemorrhage, the babies birth weight, gender. Apgar score at birth, resuscitation process, Apgar score at $5 \mathrm{~min}$ after birth were from clinical records and survival state at 1 month follow up, following up at 3 years after birth were carried out by midwives in the communities' services.

\section{Results}

From Jan. 2011 to Dec. 2013 there were 104 cases of asphyxia babies. From Jan. 2014 to Dec. 2019 there were 94 cases asphyxia. The neonate pregnant wks and birth weight, the rate of severe asphyxia at birth were not of statistic difference between two groups $\left(\chi^{2}\right.$ $=2.043, P=0.153$, at five $\mathrm{min}$ the severe asphyxia rate in experimental group $4.3 \%$ (4/94) was lower than that in controlled group $13.5 \%(14 / 104$, had of statistical difference $\left(\chi^{2}=4.968, P=0.026\right.$. Controlled group had two babies died at birth, one case was 29 wks, male, birth weight $900 \mathrm{~g}$, the second case is female, $28 \mathrm{wks}$, $950 \mathrm{~g}$; experimental group had one baby died at birth, $30 \mathrm{wks}$, male, $1700 \mathrm{~g}$; the mortality rate of newborns between two groups was not of statistical difference; all babies survived at 1 month follow up, and controlled group had one baby with severe palsy at 3 years with difficult in speaking and walking, experimental group had no palsy case. There was no difference in the rate of palsy between two groups (Table 1).

\section{Discussion}

Many studies had proved that delayed umbilical cord clamping (DCC) as compared with immediate umbilical cord clamping (ICC) can reduce the risk of mortality and the need for blood transfusions $[5,6]$. It is believed the beneficial effect of DCC mainly was attributed to a larger circulating neonatal blood volume by increased placental transfusion [7].

Avoiding hypoxemia, especially in the first minutes after birth, is pivotal since this is associated with lower $\mathrm{HR}$ and increased risk of intraventricular hemorrhage and death.

And the rate of severe Apgar score at birth was not of statistical at birth in this study, and lower rate of severe asphyxia at $5 \mathrm{~min}$ in experimental group, that may be attributed to the continuous supplication of blood and oxygen from placenta transfusion. Also the palsy rate between two groups was not of difference, the benefit of resuscitation with intact cord should be considered.

\section{Conclusions}

Resuscitation with intact cord improves the Apgar score at $5 \mathrm{~min}$ without increasing the death rate and palsy rate. It should be considered in clinical practice and larger sample of observation study should be needed for more solid results.

\section{Declaration}

We declare that there is no conflict of interest.

\section{References}

1. Li N, Yang LC, Wu Q, Han CC, Wang L, et al. (2012) The effects of iron stores and growth of delayed umbilical cord clamp timing on term breastfed infants at 4 months. Zhonghua Yu Fang Yi Xue Za Zhi 46: 303-306.

2. Scheans $P$ (2013) Delayed cord clamping: A collaborative practice to improve outcomes. Neonatal Netw 32: 369-373.

3. Menget A, Mougey C, Thiriez G, Riethmuller D (2013) Advantage of delayed umbilical cord clamping in the newborn infant. Arch Pediatr 20: 1022-1027.

4. Brouwer E, Knol R, Vernooij ASN, van den Akker T, Vlasman 
PE, et al. (2018) Physiological-based cord clamping in preterm infantsusing a new purpose-built resuscitation table: A feasibility study. Arch Dis Child Fetal Neonatal Ed 104: 396-402.

5. Fogarty M, Osborn DA, Askie L, Seidler AL, Hunter K, et al. (2018) Delayed vs early umbilical cord clamping for preterm infants: A systematic review and meta-analysis. Am J Obstet Gynecol 218: 1-18.
6. Rabe H, Diaz-Rossello JL, Duley L, Gyte GM (2012) Effect of timing of umbilical cord clamping and other strategies to influence placental transfusion at preterm birth on maternal and infant outcomes. Cochrane Database Syst Rev 9: CD003248.

7. Katheria AC, Lakshminrusimha $S$, Rabe $H$, McAdams R, Mercer JS (2017) Placental transfusion: A review. J Perinatol 37: 105-111. 